\title{
SCC Monitoring of Chloride Droplets on Thin SUS304 Plate Specimens by Analysis of Continuous Recorded AE Waveform*1
}

\author{
Kaita Ito $^{1, * 2}$, Hisashi Yamawaki ${ }^{2}$, Hiroyuki Masuda ${ }^{2}$, Mitsuharu Shiwa $^{2}$ and Manabu Enoki ${ }^{1}$ \\ ${ }^{1}$ Department of Materials Engineering, The University of Tokyo, Tokyo 113-8656, Japan \\ ${ }^{2}$ National Institute for Materials Science, Tsukuba 305-0047, Japan
}

\begin{abstract}
An acoustic emission (AE) monitoring method for chloride stress corrosion cracking (SCC) in SUS304 stainless steel has been proposed to investigate this mechanism. The method combines a droplet SCC monitoring system and an AE system capable of continuous waveform data acquisition and storage. $\mathrm{MgCl}_{2}$ droplets were placed on a thin plate specimen of SUS304 to induce SCC. Since detected AE waveforms had small amplitudes with large noises, a means of noise filtering was developed to extract these features, based on time-frequency analysis of the continuous waveform. About $200 \mathrm{AE}$ events were taken out after filtering. SEM observation showed that the AE source was attributable to transgranular SCC which cannot be detected by conventional AE monitoring systems. Therefore the new method demonstrated high sensitivity for the SCC monitoring. [doi:10.2320/matertrans.I-M2010813]
\end{abstract}

(Received December 2, 2009; Accepted May 19, 2010; Published July 7, 2010)

Keywords: stress corrosion cracking, droplet, acoustic emission method, continuous recording, time-frequency analysis

\section{Introduction}

Stress corrosion cracking (SCC) is known as one of the main causes of the fracture of metals. However, several parts of the generation mechanism of SCC still remain unclarified. This is a severe problem and early clarification is desired for industrial equipments on seaside areas, especially stainless steel which is frequently used with paintless or similar surface conditions, because chloride ion in the sea salt particles strongly induces SCC.

In this and our previous research, ${ }^{1)}$ a droplet SCC testing system $^{2)}$ which was developed by Masuda was used. This system enables a test with droplet-attached specimen in an elastic tensile stress, i.e. a more practical condition than the case of conventional U-bend specimens in solution. Furthermore, this system enables quick and highly reproducible generation of SCCs and direct observation on the surface of the specimen after the tests with high magnification. Consequently, the clarification of the mechanism of SCC with nano electrochemical approaches is advanced by this system and super Kelvin force microscope (SKFM), which provides wide area photograph and surface potential map ${ }^{3)}$ simultaneously.

Acoustic Emission (AE) method is known as a unique in-situ method to detect the generation and extension of microfractures in materials. ${ }^{4)}$ The detail of measurement of SCC with AE method was already reported by Takemoto's group. ${ }^{5-7)}$ They reported a good correspondence between the corrosion potential fluctuation and $\mathrm{AE}$, and an estimation of the cause and size of SCC by AE source wave analyses. However, they also reported that $\mathrm{AE}$ events were detected from intergranular SCCs, however, no AE events was detected with transgranular SCCs without pit because the AE signal from micro extension of SCC would be very small. $5,8,9)$

\footnotetext{
${ }^{*} 1$ This Paper was Originally Published in Japanese in J. JSNDI 58 (2009) 25-29.

${ }^{* 2}$ Graduate Student, The University of Tokyo. Present address: National Institute for Materials Science, Tsukuba 305-0047, Japan
}

Consequently, a useful monitoring method to clarify the generation mechanism of SCC, which uses the previously described droplet SCC testing system and a continuous AE measurement system which was developed by Ito $^{10,11)}$ is suggested in this research. This AE measurement system records the whole waveform during the testing time to make analyses with optimal noise filter and detection conditions of AE events which are based on observation and understanding of the characteristics of actual waveform after the test. Therefore, this system detects small AE events easier than the conventional systems which detect only AE events which satisfied the preset requirements which are based on predictions or try-and-error results. The test system and AE analysis methods were improved from our previous report. ${ }^{1)}$

\section{Measurement Method}

\subsection{Droplet SCC test system}

Figure 1 shows the developed droplet SCC test system. The specimen was a thin plate with thickness of $0.05 \mathrm{~mm}$ and $0.2 \mathrm{~mm}$, which is elastically deformed in $\mathrm{U}$ shape and fixed at the both ends. A droplet was placed on the center of the specimen. This method with $U$ shaped specimen is superficially similar to the conventional U-bend method. However, our method was significantly improved at two points. At first, the thin specimen did not require special tools for setup and it made the tests easier than the thick U-bend specimen with $15 \mathrm{~mm}(\mathrm{~W}) \times 70 \mathrm{~mm}(\mathrm{~L}) \times 2 \mathrm{~mm}(\mathrm{~T})$ size and $7.5 \mathrm{~mm}$ of curvature radius. Second, the environment of the test was more practical than the U-bend method because the specimen was deformed elastically, not plastically. Furthermore, the specimen would back to a flat plate when it was detached after the test. It is convenient to make an observation specimen for microscopes such as SKFM and atomic force microscope.

\subsection{Continuous AE waveform measurement}

Figure 2 shows the frame format of "Continuous Wave Memory" (CWM), a continuous measurement and analysis system of $\mathrm{AE}$ waveform. CWM recorded waveforms con- 


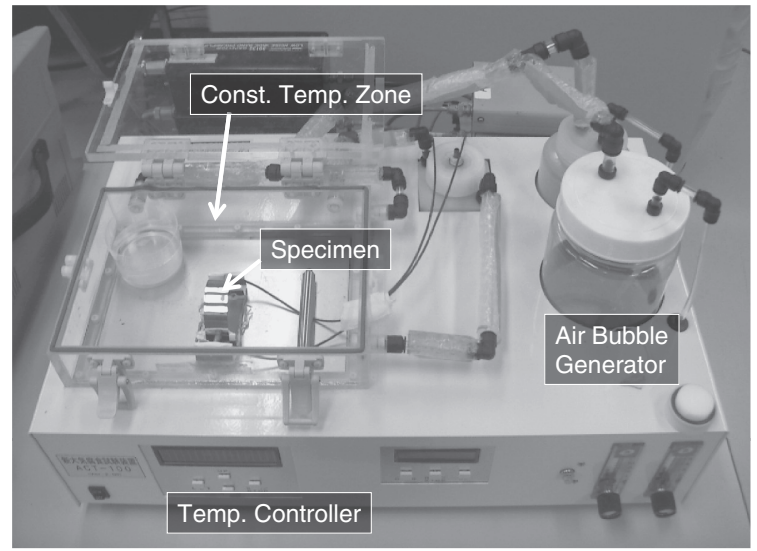

Fig. 1 SCC measurement system.

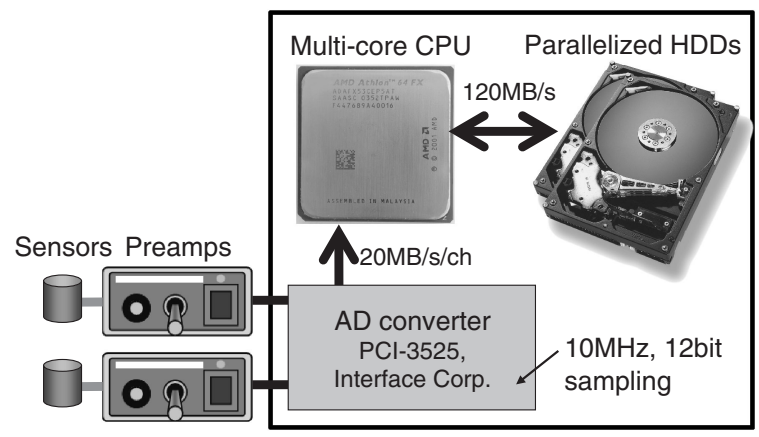

Fig. 2 Frame format of continuous wave memory (CWM).

tinuously into hard disks with $10 \mathrm{MHz}$ of sampling frequency by high speed analog-to-digital (AD) converters (PCI-3525, Interface Corp.). The recorded waveforms can be analyzed by the built-in software of CWM such as noise filtering, detection of $\mathrm{AE}$ events and calculation of $\mathrm{AE}$ parameters. CWM enabled detail estimation of AE behavior by multiple analyses with different conditions from one measurement result. Repetition of the same experiments was not needed. Furthermore, the built-in software of CWM corresponded for parallel operation with multiple CPU, hard disks and AD converters for quick analyses of multi-channel waveforms. Real time analyses have been enabled by CWM if the calculation amounts are smaller than the hardware abilities.

CWM enabled a sharp cutoff frequency filter. In this filtering process, a continuous waveform was time-frequency analyzed by short time Fourier transform (STFT) method, the rejectable frequency components were zeroed and the continuous waveform was reconstructed by inverse STFT. ${ }^{10)}$ However, some of gaps would be generated at the ends of each Fourier transform sections if inverse STFT was simply conducted. Then, a smoothing operation which used coefficient of the Hanning window function (1) was conducted.

$$
h(m)=0.5-0.5 \cos (2 \pi m / n) \quad 0 \leq m<n
$$

where $h(m)$ is the coefficient of Hanning window function, $n$ is the window length of Fourier transform, $m$ is the position in a window. $h(m)$ was applied to two streams of the results of inverse STFT which had $\mathrm{n} / 2$ shift on the position of window like below.

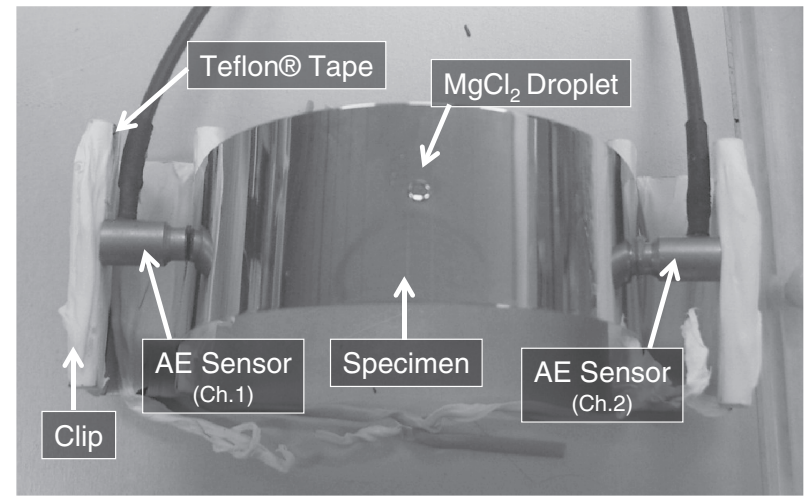

Fig. 3 SUS304 specimen on the droplet SCC measurement system.

$$
\begin{array}{r}
s(m)=s_{1}(m) h(m)+s_{2}\left(m+\frac{n}{2}\right) h\left(m+\frac{n}{2}\right), \\
0 \leq m<n / 2
\end{array}
$$

where $s_{1}$ and $s_{2}$ are continuous waveforms by inverse STFT and $s_{1}(m)$ and $s_{2}(m+n / 2)$ point the same time. Consequently, a continuous wave with no gap and small fluctuation of amplitude was obtained because $h(m)$ approached zero at the ends of windows and $h(m)+h(m+n / 2)$ was always 1 .

\section{Experimental Method}

SCC test and AE measurement of stainless steel specimen were conducted by the developed SCC test system and the continuous AE waveform measurement system "CWM". Figure 3 shows the settlement of the specimen. The specimen was a SUS304 thin plate with $20 \mathrm{~mm}(\mathrm{~W}) \times 100 \mathrm{~mm}(\mathrm{~L}) \times$ $0.2 \mathrm{~mm}$ ( $\mathrm{T}$ ) size and hardness of $\mathrm{Hv} 420$. This specimen was set on the SCC test system and bent with a stress which was close to the elastic limit. In this condition, a $3 \mu \mathrm{l}$ of $25 \%$ $\mathrm{MgCl}_{2}$ droplet was dropped on the tensile surface, i.e., the upper surface of the specimen. The temperature and humidity were kept $343 \mathrm{~K}$ and $30 \%$, respectively. These temperature and humidity kept the concentration of $\mathrm{MgCl}_{2}$ constant. Two of $200 \mathrm{kHz}$ resonant type AE sensors (M204A, Fuji Ceramics $)^{12)}$ were attached at the both ends of the specimen. These sensors enabled high sensitivity by internal head amplifier. The output signals from these sensors were amplified $45 \mathrm{~dB}$ by pre-amplifiers (A1001, Fuji Ceramics) and continuously recorded by CWM with $2 \mathrm{MHz}$ sampling frequency and 12 bit resolution. Teflon ${ }^{\circledR}$ tape was bound between the clip and the specimen to reduce the friction noise which was detected from the fixing point of the specimen during the first time tests.

\section{Results}

Figure 4 is a laser microscope photograph of the surface of the SUS304 specimen after the SCC test. A SCC with total $1 \mathrm{~mm}$ of length, right and left $500 \mu \mathrm{m}$ each was observed from the center pit with $40 \mu \mathrm{m}$ of diameter. A scanning electron microscope (SEM) photograph of the fracture surface at the tip of SCC is shown in Fig. 5. Elongated fracture surfaces by transgranular cracks with several micro- 


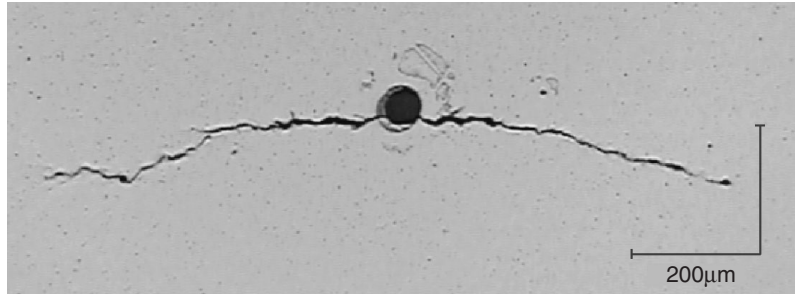

Fig. 4 Surface observation by laser microscope.

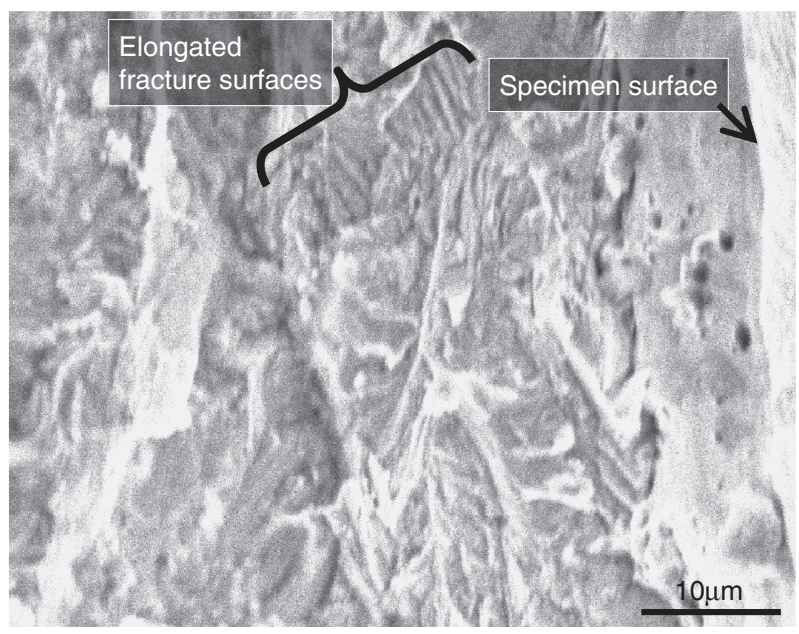

Fig. 5 SEM observation near SCC tip.

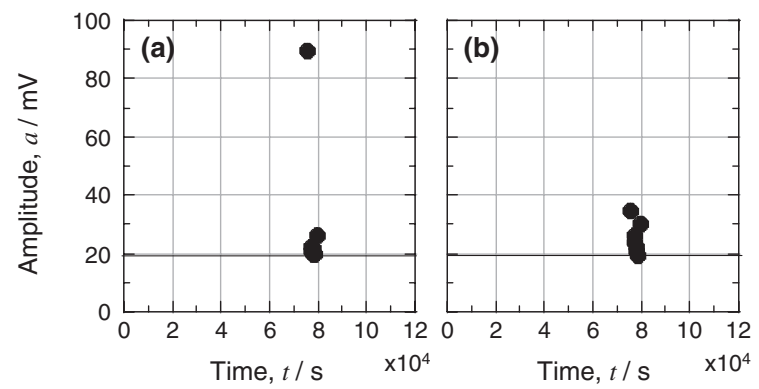

Fig. 6 AE events of the continuous waveform with $20 \mathrm{mV}$ threshold voltage, (a) ch. 1 and (b) ch. 2.

meters intervals were observed up to the depth about $30 \mu \mathrm{m}$. These transgranular cracks were observed all around the SCC.

Figure 6 shows the detection time and the maximum amplitude of $\mathrm{AE}$ events which were detected from the as-recorded continuous waveform without noise reduction process. However, type A1001 pre-amplifier included a high pass filter (HPF) with $10 \mathrm{kHz}$ of cutoff frequency. Such HPF is generally included in AE pre-amplifiers to cut low frequency noises from experimental equipments and surrounding environment and it does not affect on the $\mathrm{AE}$ wave from frequency tens to hundreds of kilohertz. A threshold voltage to detect $\mathrm{AE}$ events was set as $20 \mathrm{mV}$, which is equal to $40 \mu \mathrm{V}$ of input signal to the pre-amplifiers. This value was selected as the lowest value not to misidentify background noise to an AE event. For this purpose, a contrast test without droplet was conducted to measure the noise level and then a slightly higher value than the noise level was set as the
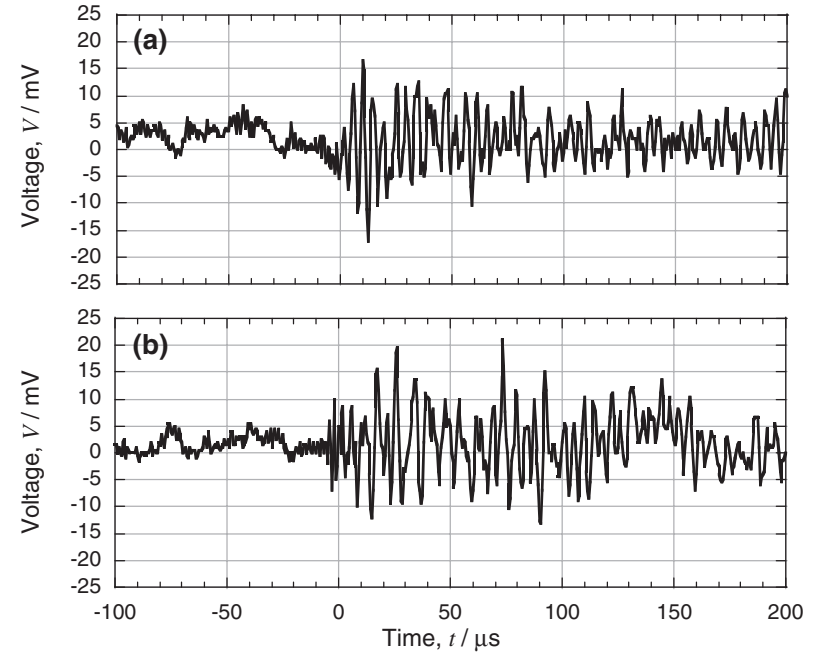

Fig. 7 AE waveform of burst type event, (a) ch. 1 and (b) ch. 2.
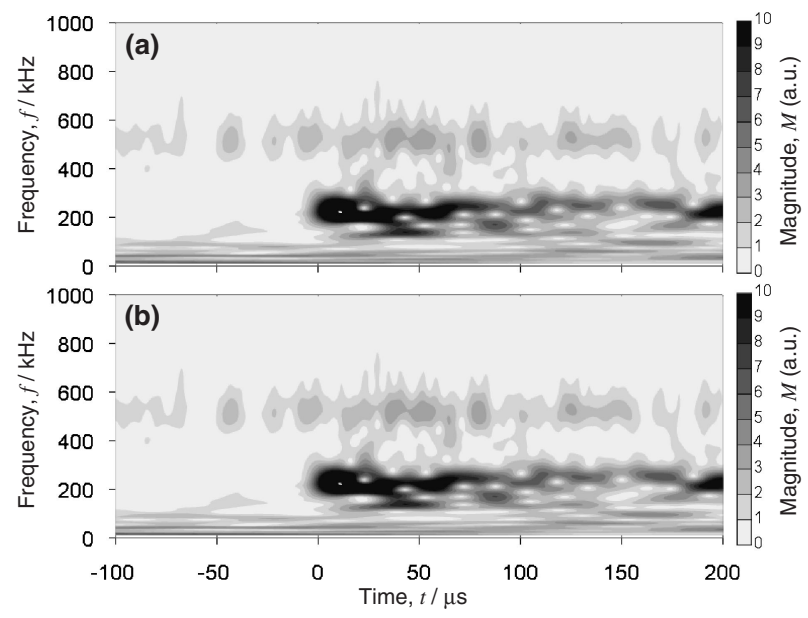

Fig. 8 Wavelet transform of AE waveform shown in Fig. 7, (a) ch. 1 and (b) ch. 2 .

threshold level. As a result, several burst AE events with waveforms like in Fig. 7 were detected. However, the number of AE events was only seven.

Consequently, the frequency filter was applied to the continuous waveform by CWM as a noise reduction process to improve the detection sensitivity of AE events. Figure 8 shows the spectrogram of the waveform in Fig. 7. However, Gabor wavelet transform was used instead of STFT to estimate the short time changes around the occurrence time of the AE event. Then, intensity peaks which were constantly observed before the occurrence time of the AE event, i.e., the background noise components were observed under $100 \mathrm{kHz}$ and over $500 \mathrm{kHz}$. At the same time, another intensity peak was observed only after the occurrence time of the AE event, i.e. the AE event itself was observed between around 100 and $200 \mathrm{kHz}$, and it was near to the resonant frequency of the AE sensors. Based on these signal characteristics, a band pass filter (BPF) from 100 to $300 \mathrm{kHz}$ was applied to the continuous waveform to keep the signal component of $\mathrm{AE}$ event and eliminate the noise component. Then, $4 \mathrm{mV}$ of threshold voltage which is equal to $8 \mu \mathrm{V}$ of input signal to the pre-amplifiers was set to the filtered waveform and about 200 

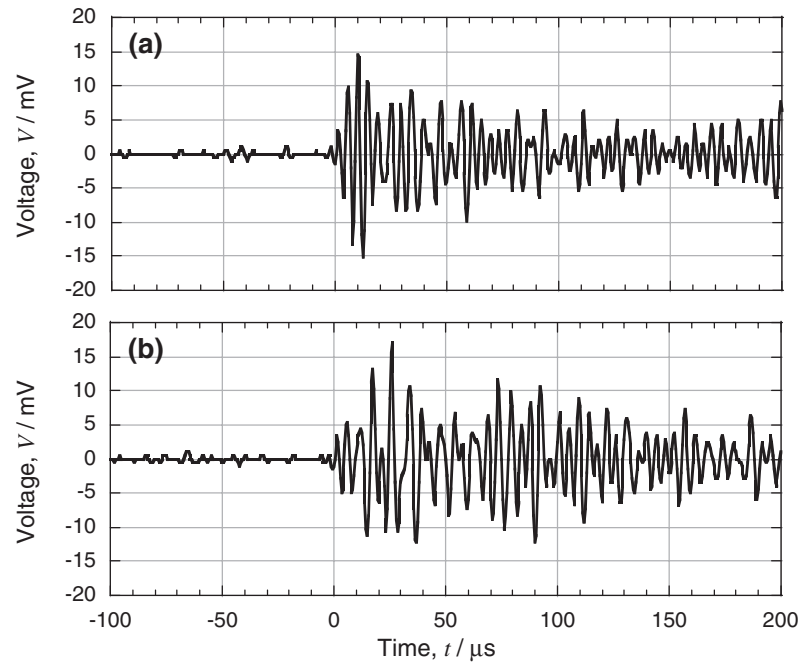

Fig. 9 Noise reduced AE waveform shown in Fig. 7, (a) ch. 1 and (b) ch. 2.

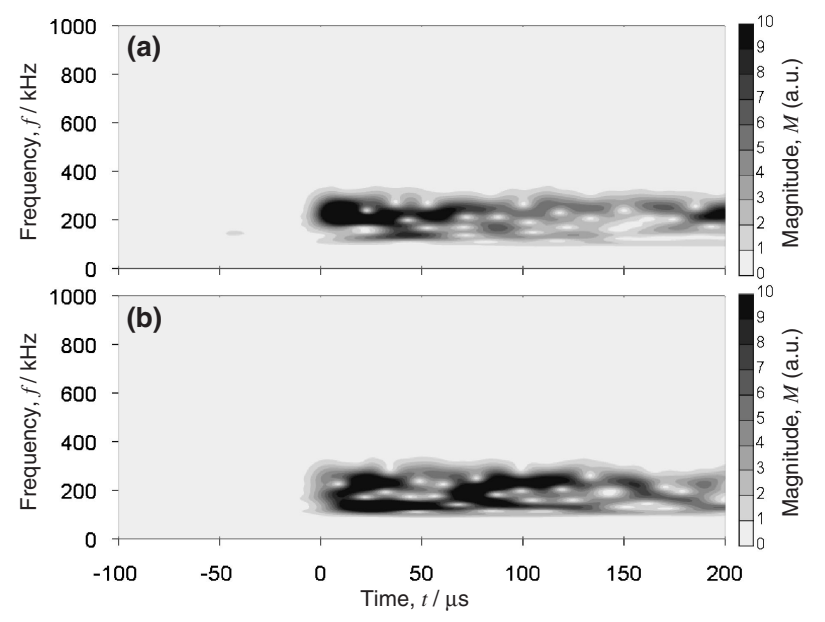

Fig. 10 Wavelet transform of AE waveform shown in Fig. 9, (a) ch. 1 and (b) ch. 2 .

$\mathrm{AE}$ events were detected. It was a lower threshold voltage than the previous case of non-filtered waveform. Figures 9 and 10 show the waveform and the spectrogram by the Gabor wavelet transform of the same AE event in Fig. 7. The BPF in CWM which used STFT showed a sharp cutoff and effective noise reduction. And then, Fig. 11 shows the occurrence times and the maximum amplitudes of AE events which were detected from the filtered waveform likes Fig. 6. A large number of AE events were detected around $7.6 \times 10^{4} \mathrm{~s}$, that is the same time as a small number of $\mathrm{AE}$ events were detected from the non-filtered waveform. In addition, large numbers of burst $\mathrm{AE}$ events with small amplitudes were newly detected around $3.5 \times 10^{4} \mathrm{~s}$ and $5.1 \times 10^{4} \mathrm{~s}$.

\section{Discussions}

CWM facilitated the optimization of the signal processing which is based on the spectrogram of noisy continuous waveform. Then the detection sensitivity of AE events was improved. In this study, the components of valid signal and noise were closely existed across the $100 \mathrm{kHz}$, the cutoff frequency, but the previously mentioned frequency filter
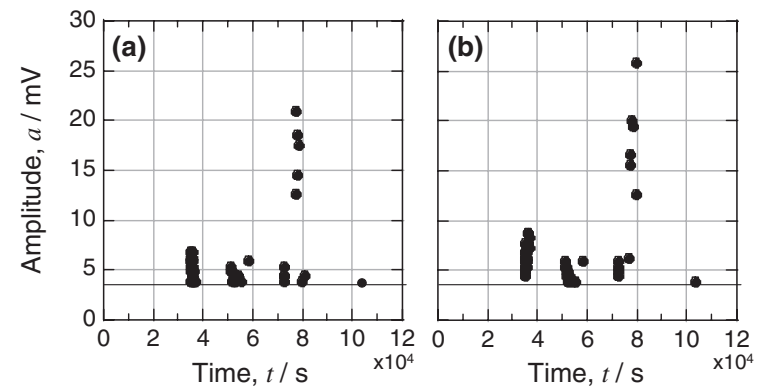

Fig. 11 AE events of the noise reduced continuous waveform with $4 \mathrm{mV}$ threshold voltage, (a) ch. 1 and (b) ch. 2.
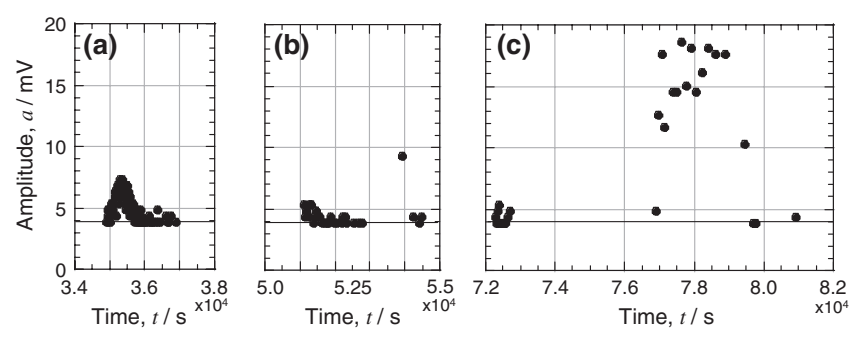

Fig. 12 Details of the $\mathrm{AE}$ events of the noise reduced continuous waveform with $4 \mathrm{mV}$ threshold voltage, (a) $3.4-3.8 \times 10^{4} \mathrm{~s}$, (b) $5.0-$ $5.5 \times 10^{4} \mathrm{~s}$ and (c) $7.2-8.2 \times 10^{4} \mathrm{~s}$.

which used STFT successfully eliminated the noise and kept the valid signal. Therefore, a larger number of AE events with small amplitudes were detected than the conventional systems.

The cause of the burst AE events which were detected in this test was presumed that the generation or migration of hydrogen or oxygen by the cathode reaction according to the corrosion reaction or plastic deformation at the crack tip. However, SEM observation of the fracture surfaces of the SCCs in this test showed only transgranular type with several micrometers of small planes and the evidence of intergranular type was not observed. Therefore, the cause of the small amplitude burst $\mathrm{AE}$ events which were detected in this test could be the extension of the transgranular SCC which could not be detected by the conventional $\mathrm{AE}$ measurement systems. In this instance, the reason of the small amplitudes of the $\mathrm{AE}$ events was presumed that the extension unit of the transgranular SCC may be several micrometers of small planes, or the generation speed of microfracture was slower than the intergranular type. Some of additional experiments are needed to clarify the mechanism of AE, for example a new test which generates an intergranular SCC with smaller stress by the same experimental equipment and in-situ observation of the SCC extension by video microscope.

As shown in Fig. 11, AE events were detected periodically and intensively in several times. It supposed that extensions and stops are repeated by the transgranular SCC like the intergranular SCC. ${ }^{13,14)}$ Extended figures of Fig. 11 around the times when $\mathrm{AE}$ occurred frequently, such as $3.5 \times 10^{4}$, $5.1 \times 10^{4}$ and $7.6 \times 10^{4} \mathrm{~s}$ are shown in Fig. 12. The length of each part is several hundred or thousand seconds and the amplitudes of AE events changed small, large and small again. These characteristics were the same as the intergranular SCC which were reported in the former studies. ${ }^{13,14)}$ 


\section{Conclusions}

(1) AE events due to the extension of transgranular SCC which could not be detected by the conventional $\mathrm{AE}$ measurement systems because of their small amplitude were successfully detected by the original SCC test system and AE waveform measurement system "CWM". It is expected that the combination of this method and other estimation methods clarify the generation mechanism of transgranular SCC.

(2) The reason of the small amplitudes of the AE events was presumed that the extension unit of the transgranular SCC may be several micrometers of small planes which were observed at the fracture surface, or the generation speed of the microfractures was much slower than the intergranular type.

(3) Extension of the transgranular SCC repeats extensions and stops likes the case of former reported intergranular SCC.

\section{Acknowledgement}

This work is supported by Japan Society for the Promotion of Science (JSPS) KAKENHI (Grants-in-Aid for Scientific Research) 19-3682 and 20656153.

\section{REFERENCES}

1) M. Shiwa, H. Yamawaki, H. Masuda, K. Ito and M. Enoki: Strength, Fracture and Complexity 5 (2009) 109-116.

2) H. Masuda: Japanese Patent Laid-open 2006-258500 (2006). (in Japanese)

3) M. Masuda: Corros. Sci. 49 (2007) 120-129.

4) Ad Hoc Research \& Technical Committee on Acoustic Emission, Jpn. Soc. Non-Destructive Inspection: Acoustic Emission I (Jpn. Soc. Non-Destructive Inspection, Tokyo, 2006) p. 4. (in Japanese)

5) S. Fujimoto, M. Takemoto and K. Ono: J. Acoustic Emission 19 (2001) 63-72.

6) S. Fujimoto and M. Takemoto: Zairyo-to-Kankyo 53 (2004) 309-316. (in Japanese)

7) A. Yonezu, H. Cho, R. Ikeda, T. Ogawa and M. Takemoto: J. JSNDI 56 (2007) 34-40. (in Japanese)

8) H. Okada, Y. Yukawa and H. Tamura: Corrosion 30 (1974) 253-255.

9) H. Okada, Y. Yukawa and H. Tamura: Corrosion 32 (1976) 201-213.

10) K. Ito and M. Enoki: Mater. Trans. 48 (2007) 1221-1226.

11) K. Ito and M. Enoki: J. Japan Inst. Metals 71 (2007) 1061-1065. (in Japanese)

12) M. Shiwa, H. Inaba and T. Kishi: Mater. Evaluation 50 (1992) 868874.

13) T. Kishi: Proc. 78th 79th Nishiyama Memorial Tech. Seminar (1991) pp. 133-170. (in Japanese)

14) S. Yuyama, T. Hisamatsu and T. Kishi: J. Japan Inst. Metals 46 (1982) 85-93. (in Japanese) 